\title{
QoS ROUTING USING GENETIC ALGORITHM (QOSGA)
}

\author{
R. Leela and S. Selvakumar
}

\begin{abstract}
The current Internet architecture supports best-effort data delivery by default, which has provided satisfactory services for various applications, such as the email and file transfer, to a great extent. On the other hand, the increase in real-time multimedia applications such as Voice over IP, audio and video streaming in the public Internet demand for a Quality of Service $(\mathrm{QoS})$ routing that satisfies multiple constraints such as bandwidth, delay, delay jitter, packet loss, cost, etc. To find a feasible path satisfying multiple constraints is NP-complete [2]. Hence the recent researches on QoS based routing have triggered the proposition of many heuristic QoS routing algorithms [1]-[4], [15], [16]. The time taken by these heuristics to find a feasible path is high [4]. To search all feasible paths in less time, many researchers have used the concept of Genetic Algorithm (GA), which is a new computational strategy inspired by natural processes. The aim of these routing algorithms is to aid the fast selection of a feasible path, which should be adaptive, flexible, and intelligent for efficient network management. The focus of this paper is to develop a GA based routing algorithm that satisfies multiple constraints requirement of the multimedia applications. Hence, in this paper a heuristic called QoS ROUTING USING GENETIC ALGORITHM (QOSGA), which incorporates multiple constraints required by multimedia applications to find a feasible path, has been proposed, designed, and simulated. The processing time taken by the proposed algorithm has been compared with the existing non-GA based heuristic Self Adaptive Multi-Constrained Routing Algorithm (SAMCRA). Also, the number of generations taken by QOSGA to find a feasible path is compared with the number of generation taken by the GA based algorithms1 Genetic Load Balancing Routing Algorithm (GLBR) and Adaptive Routing method based on Genetic Algorithm with two QoS constraints (ARGAQ). The results confirm that QOSGA performs better in terms of time taken to return feasible paths.
\end{abstract}

\section{Index Terms- QoS, SAMCRA, Genetic Algorithm.}

\section{INTRODUCTION}

The current Internet is essentially a connection-less network, which supports best effort traffic. The data packets may follow different paths to the destination. The network resources, e.g., switch buffer and link bandwidth, are fairly shared by packets from different sessions [18]. While this type of network is suitable for current applications such as email, ftp, etc., it would be intolerable for upcoming real time multimedia applications such as video conferencing, Internet telephony, and video-on-demand. The two primary

$\mathrm{R}$, Leela is with the Department of CSE, National Institute of Technology, Tiruchirappalli, TamilNadu, India.

S. Selvakumar is with the Department of CSE, National Institute of Technology, Tiruchirappalli, TamilNadu, India. reasons for not supporting the multimedia applications are the following: First, it does not support resource reservation, which is vital, for the delivery of guaranteed end-to-end performance (bounded delay, bounded delay jitter, and/or bounded loss ratio). Second, data packets may experience unpredictable delays and arrive at the destination out of order, which is undesirable for continuous real-time media [15], [19]. These requirements of the application force the network service provider to guarantee QoS. QoS refers to the collective effect of service performance that determines the degree of satisfaction of a user of the service [18]. QoS routing is to find a feasible path that meets specific QoS requirements.

The problem of QoS routing is, however, not a trivial one, due to the following reasons [15]: First, the distributed applications such as Internet phone and distributed games demand very diverse QoS constraints on delay, delay jitter, cost, loss ratio, bandwidth, etc. Multiple constraints often make the routing problem intractable. For example, finding a feasible path with two independent path constraints is NP-complete [16]. Second, the network state changes dynamically due to transient load fluctuations, connections in and out, and links going up and down. The growing network size makes it increasingly difficult to gather up-to-date state information in a dynamic environment. The performance of a QoS routing algorithm can be seriously degraded if the state information being used is outdated. Third, any future integrated-service network is likely to carry both QoS traffic and best-effort traffic, which makes the issue of performance optimization complicated. It is hard to determine the best operating point for both types of traffic if their distributions are independent. Although the QoS traffic will not be affected due to resource reservation, the throughput of the best-effort traffic will suffer if the overall traffic distribution is a misjudge. Fourth, today's optimal path routing algorithms do not support alternate routing. If the best existing path cannot admit a new flow, the associated traffic cannot be forwarded even if an adequate alternate path exists. Hence it is evident that the QoS routing algorithms must be adaptive, flexible, and intelligent enough to make a fast decision. To achieve this, a Genetic Algorithm (GA) that uses the computational strategies inspired by natural processes is used. GA is a global optimization technique derived from the principle of nature selection and evolutionary computing or technique [9], [12], [14], [17]. GA has been theoretically and empirically proven to be robust search technique. Each possible point in the search space of the problem is encoded into a representation suitable for applying GA. GA transforms a population of individual solutions, each associated with a fitness value 
into a new generation of the population, using the Darwinian principle of the survival of the fittest. By applying genetic operators such as crossover and mutation, GA produces better approximations to the solutions. At each iteration, the process of selection and reproduction creates a new generation of approximations. Figure 1 represents the outline of the basic GA.

Many GA based routing algorithms have been proposed. In this paper, a study of the existing non-GA based [1]-[4], [15], [16] and GA based QoS routing algorithms [10]-[13] has been done. The drawback in all these existing algorithms has been the time taken to find a feasible path and the consideration of single or single mixed constraint while computing the feasible path. This has been the motivation to propose a new heuristic QOSGA that satisfies multiple constraints for finding a feasible path and apply GA to reduce the time taken to find a feasible path, in this paper. The rest of the paper is organized as follows: Section II describes the composition rules for QoS constraints. Section III defines the problem statement. Section IV discusses the existing non-GA based and GA based QoS routing algorithms. The new heuristic QOSGA that considers multiple constraints for routing decision has been proposed in Section V. The simulation results of QOSGA are discussed in Section VI and finally Section VII summarizes the paper.

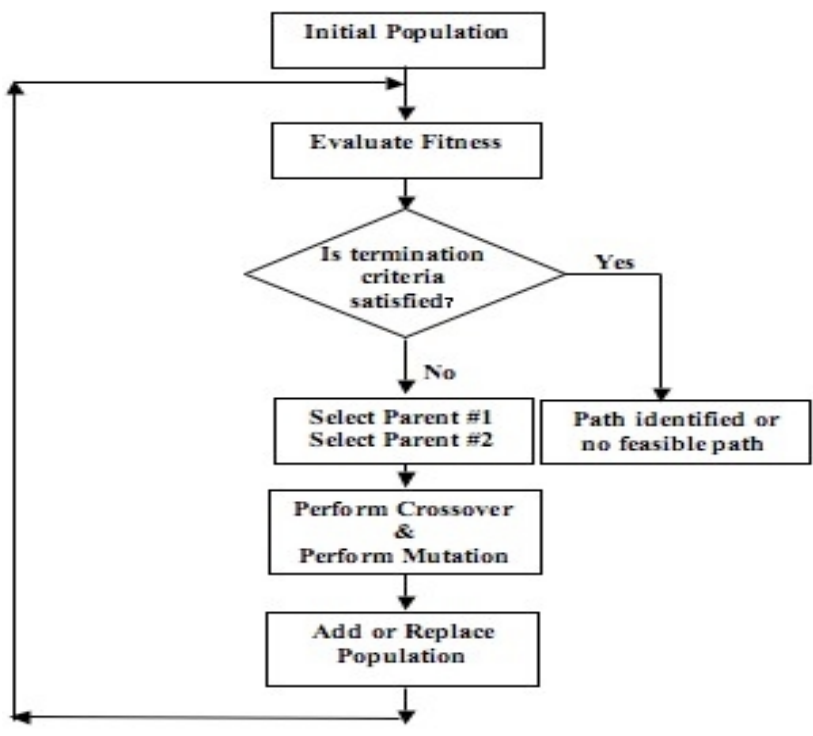

\section{QoS Constraints}

The QoS requirement of an application is given as a set of constraints, which can be link constraints or path constraints A link constraint specifies the restriction on the use of links. For instance, a bandwidth constraint of a unicast connection requires that the links composing the path must have certain amount of free bandwidth available. A path constraint specifies the end-to-end QoS requirement on a single path [18]. Each link in the network is associated with multiple parameters that can be roughly classified into additive and non-additive constraints.

\section{A. Additive Constraints}

It can be represented mathematically as follows:

$$
m(p)=\sum_{i=1}^{L K} m\left(l k_{i}\right)
$$

where $m(p)$ is the total of metric m of path $(p), l k_{i}$ is the link $\mathrm{i}$ in the path $(p), m\left(l k_{i}\right)$ is the metric in link $\mathrm{i}, L K$ is the number of links in path $(p)$, and $\mathrm{i}=1, \ldots, L K$

Examples: Delay, Delay variation (jitter), and Cost.

\section{B. Concave/convex Constraints}

It can be represented mathematically as follows:

$$
m(p)=\min / \max \left(m\left(l k_{i}\right)\right)
$$

Examples: Bandwidth (here bandwidth is the residual bandwidth that is available for network flow). It can be defined as the minimum of the residual bandwidth of all links on the path or bottleneck bandwidth.

\section{Multiplicative Constraints}

It can be represented mathematically as follows:

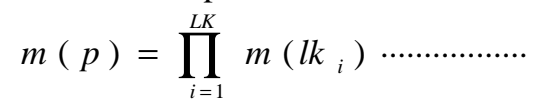

Example: Loss probability (indirectly). Loss probability can be easily transformed into an equivalent metric that follows the multiplicative composition rule. Probability of successful transmission can be expressed as

$S t(l k)=1-l(l k)$.

Fig. 1. Outline of the basic Genetic Algorithm

$S t(p)=\prod_{i=1}^{L K} S t\left(l k_{i}\right)$

Therefore the loss probability metric $(l)$ can be expressed mathematically as follows:

$l(p)=1-\left\{\left[1-l\left(l k_{1}\right)\right]\left[1-l\left(l k_{2}\right)\right], \ldots,\left[1-l\left(l k_{L K}\right)\right]\right\}$.

Where $S t(l k)$ is the probability of successful transmission for link $l k, S t(p)$ is the probability of successful transmission for the entire path $p, S t\left(l k_{i}\right)$ is the probability of successful transmission for link $i$ in the path $p, l(l k)$ is the loss probability for link $l k, l(p)$ is the loss probability for the entire path $\mathrm{p}$, and $l\left(l k_{i}\right)$ is the loss probability for link $\mathrm{i}$ in the path $\mathrm{p}$.

\section{Problem Statement}

Given an undirected graph $\mathrm{G}(\mathrm{N}, \mathrm{E})$, where $\mathrm{N}$ is the set of nodes and $\mathrm{E}$ is the set of Edges. Each link $\mathrm{u} \rightarrow \mathrm{v} \in \mathrm{E}$ is associated with link weights $\mathrm{w}_{\mathrm{i}} \quad(\mathrm{u} \rightarrow \mathrm{v}) \geq 0$, for all $\mathrm{i}=1, \ldots$, $\mathrm{m}$. Given $\mathrm{m}$ constraints $\mathrm{L}_{\mathrm{i}}$, where $\mathrm{i}=1, \ldots \ldots, \mathrm{m}$, the problem is to find a path $\mathrm{p}$ from source node $\mathrm{s}$ to destination node $\mathrm{t}$ such that:

$\mathrm{W}_{\mathrm{i}}(\mathrm{p})=\sum_{(\mathrm{u} \rightarrow \mathrm{v}) \in \mathrm{p}} \mathrm{Wi}_{\mathrm{i}}(\mathrm{u} \rightarrow \mathrm{v}) \leq \mathrm{L}$, for all $\mathrm{i}=1, \ldots \ldots \mathrm{m} \ldots(7)$

A path satisfying the above equation (7) is said to be a feasible path. There may be a set of feasible path satisfying the constraints.

\section{Existing Methods}

There are many heuristic for QoS routing with and without using GA, proposed by recent researchers. In this section, a brief description on QoS Routing algorithms without using GA is given followed by QoS routing algorithm using GA have been briefly explained. 


\section{A. Non GA based QoS Routing Algorithms}

The problem of QoS routing has been addressed in the literature and many path computation algorithms [1]-[4], [8], [15], [16] satisfying the QoS demands of the applications have been proposed. These algorithms have been shown to be NP-complete problems. Jaffe's algorithm [2] applies Dijkstra's shortest path algorithm using a linear combination of two link weights and finds a feasible path. The main drawback of this algorithm is that the shortest path does not necessarily satisfy all the constraints. Iwata's algorithm [7] first computes one shortest path based on one QoS metric and then checks if all other constraints are met. If this condition is not satisfied, the procedure is repeated until a feasible path is found. One major drawback of this fallback approach is that there is no guarantee that optimizing path selection with respect to any single metric would lead to a feasible path. A Tunable Accuracy Multi-Constrained Routing algorithm (TAMCRA) [5] is based on the following three fundamental concepts: A non-linear measure of the path length, k-shortest path approach, and Principle of non dominated paths. A non linear measure for the path length $1(\mathrm{p})$ is given in (8). $l(p)=\max _{i=1, . ., m}\left(w_{i}(p) / L_{i}\right)$.

Where, the path vector $\mathrm{w}_{\mathrm{i}}(\mathrm{p})$ is the $\mathrm{i}^{\text {th }}$ weight path vector for the path $p$ and $L_{i}$ is the $i^{\text {th }}$ constraint. If $l(p)>1$ then the path is not feasible, else it is feasible. TAMCRA uses the $\mathrm{k}$-shortest path approach, where the user predefines $\mathrm{k}$. The parameter $\mathrm{k}$ is fixed in TAMCRA. Self Adaptive Multi-Constrained Routing Algorithm (SAMCRA) [2], which is the successor of TAMCRA, also follows these three fundamental concepts of TAMCRA and includes a fourth concept 'look ahead'. In contrast to TAMCRA, where the value of $\mathrm{k}$ is fixed, the value of $\mathrm{k}$ increases exponentially in worst case in SAMCRA. The queue size can be adapted independently at each node during the path computation instead of requiring queue size of kmax in each node. The advantage of SAMCRA over TAMCRA is that the search space is reduced by pre computing the shortest path to the destination. The link-DIsjoint Multiple Constraints Routing Algorithm DIMCRA [6] uses SAMCRA to find $k$ feasible paths and selects two shortest paths whose links are disjoint. The drawback of all these algorithms is the time taken to find a feasible path is considerably high

\section{B. GA based QOS Routing Algorithms}

1) Genetic Load Balancing Routing Algorithm (GLBR):

GLBR algorithm [13] uses gene coding in which the genes are put in a chromosome in the same order as that of the nodes forming the communication route. The algorithm starts with an identification of initial population chosen randomly. Then path genetic operations such as path crossover and path mutation are performed. Path crossover operation randomly selects a node as a crossing site and then exchanges the sub paths among the pair of paths. Path mutation operation randomly selects a node (mutation node) and finds a path to the destination by mutating using Dijkstra's shortest path algorithm. These path genetic operations are repeated until a feasible solution is found or the generation number exceeds a certain limit.

The main aim of this algorithm is to balance the load in the network. To achieve load balancing source periodically sends the delay query packet and checks whether the path is loaded or not. If the response to this query packet from the destination exceeds a certain specified threshold value, the path is recomputed, by repeating the path genetic operations. The effectiveness of the GLBR algorithm is shown in [13] by comparing it with conventional Shortest Path First (SPF) and RIP algorithms. GLBR algorithm performs better than SPF and RIP algorithms. However GLBR algorithm computes path based on a single metric (Delay Time), which is not suitable for QoS routing. Moreover, the delay query packet sent periodically may overload the network. Also, the individuals of a population have different sizes resulting in a complicated crossover operation and the genetic operations sometimes may result in a communication route with loops. Hence a check condition has to be invoked to validate each and every new path identified, which results in computation overhead.

2) Adaptive Routing method based on Genetic Algorithm (ARGA):

In order to simplify the genetic operations of GLBR algorithm and to overcome the looping problem, ARGA was proposed [10]. It uses a different kind of gene coding. This type of coding ensures that the length of each chromosome is fixed and equal. The network is expressed as a tree network, the genes are expressed as the tree junctions, and the genetic operations are carried out in the tree junctions. Since the genetic operations are performed on a tree network the identified routes are always loop free. Therefore, there is no need to check the validity of the searched routes as in GLBR algorithm. It has been shown that the ARGA performs better than GLBR algorithm in terms of faster routing decisions making. But ARGA uses only the delay time as a parameter for routing, which is insufficient for satisfying multimedia applications.

3) Adaptive Routing method based on Genetic Algorithm with two QoS constraints (ARGAQ):

In order to support multimedia applications over high-speed networks, it was necessary to develop routing algorithms [10], [12], which compute path subject to more than one QoS constraint. Hence ARGA was enhanced to ARGAQ, which considered two QoS constraints as a single mixed metric. ARGAQ computes path subject to the two constraints such as Delay Time and Transmission Success Ratio (TSR). TSR is the rate of packets transmitted without loss. Except the fitness evaluation step, all the steps of ARGAQ are the same as ARGA. The fitness value T of each individual path is calculated using the following equation:

$$
\mathrm{T}=\frac{\sum_{\mathrm{i}=1}^{\mathrm{n}} \mathrm{DT}_{\mathrm{i}}}{\prod_{\mathrm{i}=1}^{\mathrm{n}} \mathrm{TSR}}
$$

Where, $\mathrm{DT}_{\mathrm{i}}$ represents the Delay Time for the link $\mathrm{i}$ and $\mathrm{TSR}_{\mathrm{i}}$ represents the Transmission Success Ratio of the link i, and $n$ represents the total number of nodes in the path. ARGAQ provides only an approximate approach to QoS routing as it computes path subject to single mixed metric $\mathrm{T}$, which is the ratio of Delay Time and Transmission Success Ratio.

To summarize the existing GA based QoS routing algorithms, GLBR algorithm and ARGA use only single 
metric whereas ARGAQ provides an approximate approach by computing the path subject to single mixed metric. But multimedia applications require QoS route, which satisfies multiple constraints.

\section{Proposed Heuristic}

In this Section, a new heuristic called QoS Routing Using Genetic Algorithm (QOSGA) has been proposed. QOSGA is a unicast QoS source routing algorithm. The algorithm along with the justification, step-by-step procedure, constraints considered, detailed illustration, and the advantages are described in this Section.

\section{A. Algorithm}

The steps involved in the working of the proposed QOSGA are listed below:

- Use the connectivity of the network to generate the initial gene population

$$
\text { - Repeat }
$$

- Compute the value of fitness function using equation

- Rank the paths based on the value of the fitness function

(as the

- If the value of the first rank $\leq 1$ then terminate

$$
\text { path is found) }
$$

- else Use lower ranked paths to create new genes SET

- Until a path is found or the number of iterations =

\section{VALUE}

\section{B. DETAILED ILLUSTRATION}

1) Representation and Encoding

Encoding is one of the problems found when GA is used for finding a solution. Encoding depends on the problem for which GA is applied. In this paper the network is expressed by a tree network and the genes are expressed by the tree junction [10]. By this coding method the length of each chromosome is same and the genetic operations are carried out in the tree junction. To explain the encoding procedure a sample network given in Figure 2 is considered. Node 1 is the source node and 5 is the destination node. Table I gives the gene coding for the network in Figure 2.

\begin{tabular}{|c|c|c|c|c|c|c|}
\multicolumn{2}{c}{ Table I GENE CODING } \\
\hline Junction & 0 & 1 & 2 & 3 & 4 & 5 \\
\hline Gene coding & 237 & 36 & 24 & 56 & 57 & 25 \\
\hline
\end{tabular}

\begin{tabular}{|c|c|c|}
\hline \multicolumn{1}{|c|}{ Table II Initial Population with fitness value } \\
\hline Item & Path & Fitness Value \\
\hline $\begin{array}{c}\text { Chromoso } \\
\text { me } 1\end{array}$ & $1 \rightarrow 2 \rightarrow 3 \rightarrow 4 \rightarrow 5$ & 1.3 \\
\hline
\end{tabular}

\begin{tabular}{|c|c|c|}
\hline $\begin{array}{c}\text { Chromoso } \\
\text { me2 }\end{array}$ & $1 \rightarrow 3 \rightarrow 2 \rightarrow 6 \rightarrow 5$ & 1.28 \\
\hline $\begin{array}{c}\text { Chromoso } \\
\text { me3 }\end{array}$ & $1 \rightarrow 7 \rightarrow 6 \rightarrow 2 \rightarrow 3 \rightarrow 4 \rightarrow 5$ & 1.76 \\
\hline
\end{tabular}

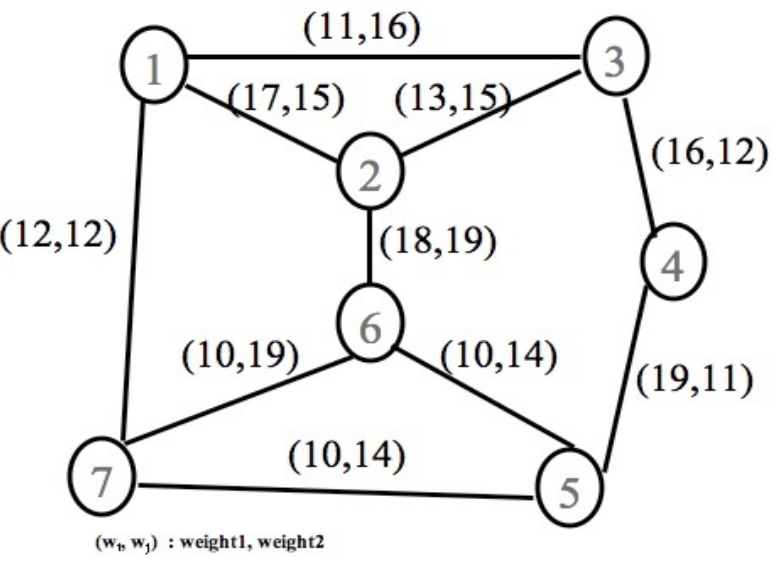

Fig. 2 Sample Network

2) Evaluation of Fitness function

The association of fitness value to each solution is done through a fitness function. The fitness function used in this paper is given in equation (10).

$$
\text { fitness value }=\max \left(\frac{\sum_{i=1}^{m} w_{i}(p)}{L_{i}}\right)
$$

Where $\mathrm{m}$ is the total number of constraints considered, $\mathrm{p}$ is the path, $\mathrm{Li}$ is the maximum acceptable constraint value specified for the application, and wi is the link weights which is static and depends on the physical characteristics of the link. In Figure 2 two weights corresponding to two parameters are assigned to each link. The constraint limit L1 and L2 is specified to be 50 each. Table II gives the initial population with the fitness value calculated for each chromosome.

3) Chromosome Selection

Chromosomes are selected from the initial population to be parents. According to Darwin's evolution theory the best one should survive and create new offspring. There are many methods available for selecting the chromosomes such as roulette wheel selection, steady state selection, tournament selection, elitism selection, etc. In this paper, the elitism selection method is used. Elitism is the method, which copies the best chromosomes to new population. The chromosomes are selected for genetic operation by sorting the chromosomes in the initial population by their fitness value and then selecting the first two in the list. Table III represents the sorted and selected list of chromosomes for the given example.

Table III Sorted and Selected Chromosomes

\begin{tabular}{|l|c|c|}
\hline \multicolumn{3}{|c|}{ Table III Sorted and Selected Chromosomes } \\
\hline Item & Path & $\begin{array}{c}\text { Fitness } \\
\text { value }\end{array}$ \\
\hline
\end{tabular}




\begin{tabular}{|c|c|c|}
\hline Chromosome2 & $1 \rightarrow 3 \rightarrow 2 \rightarrow 6 \rightarrow 5$ & 1.28 \\
\hline Chromosome1 & $1 \rightarrow 2 \rightarrow 3 \rightarrow 4 \rightarrow 5$ & 1.3 \\
\hline
\end{tabular}

\section{4) Crossover and Mutation}

Crossover and Mutation are the two basic operators of GA. Performance of GA depend on them. Implementations of these operations depend on the encoding and also on the problem for which GA is used. This paper uses single point cross over at the tree junction to generate new offspring. The mutation point selected is those points, which lead to violation of constraint satisfaction. Figures 3 and 4 illustrate the crossover and mutation operation for the sample network considered.

\section{5) Acceptance}

Add the output which is the outcome of the genetic operations to the population and repeat the process from the step that evaluates the fitness function.

\section{6) Termination}

The genetic operation is repeated until $\mathrm{k}$ feasible paths are found or the number of generation exceeds a set value. Table IV gives the first two feasible paths identified by the proposed algorithm.

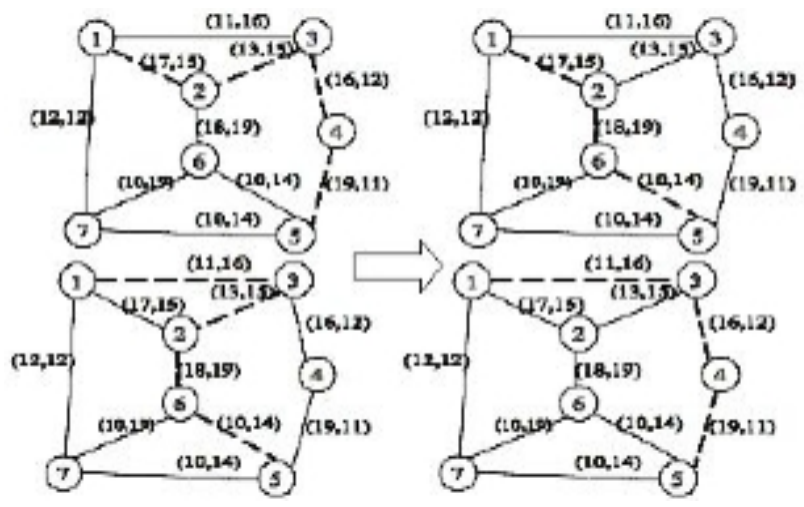

Fig. 3 Crossover Operation

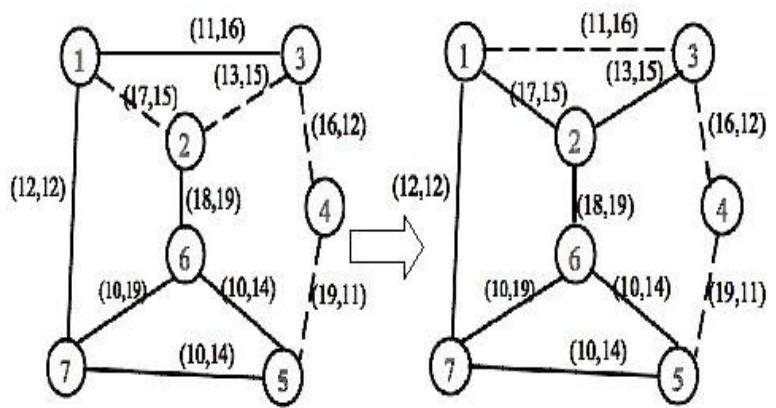

Fig. 4 Mutation Operation

Table IV Chromosome after Genetic Operatons

\begin{tabular}{|c|c|c|}
\hline \multirow{2}{*}{ Item } & Path & Fitness value \\
\hline Chromosome4 & $1 \rightarrow 2 \rightarrow 6 \rightarrow 5$ & .96 \\
\hline Chromosome5 & $1 \rightarrow 3 \rightarrow 4 \rightarrow 5$ & .92 \\
\hline
\end{tabular}

7) Path selection

For the sample network given in Figure 2 the feasible paths identified using GA are listed in Table IV. From Table IV it is observed that the path denoted by chromosome 5 has less fitness value compared to the path represented by chromosome 4 . Hence path represented by chromosome 5 will be selected for sending multimedia packets of the requested application.

\section{Simulation Result}

Comparison with non GA based QoS Routing Algorithm The QOSGA has been simulated and experimented for different networks of size ranging from 8 to 200 nodes. Each network is tested with both SAMCRA and QOSGA, and the time taken for path computation is observed. Figure 5 shows the processing time taken to find the feasible path by QOSGA and SAMCRA. As the unit of processing time is microseconds the y-axis in Figure 5 is chosen to be log scale so that the difference between the two algorithms will be distinctly clear. From Figure 5 it is evident that the time taken for finding feasible paths increases in SAMCRA and QOSGA as the network size increases. But the increase is more in SAMCRA when compared to QOSGA. The reduced time for path computation could be attributed mainly due to the usage of Genetic Algorithm and the way the genetic operators are used in order to find the feasible path quickly.

\section{A. Comparison with GA based QoS Routing Algorithm}

QOSGA has been simulated and experimented for different networks. The fitness value of the chromosome with Rank 1 versus Generation number for the algorithms GLBR, ARGAQ, and QOSGA for a network of size 200 nodes is shown in Figure 6. From Figure 6, it is evident that QOSGA finds the feasible path at 3rd generation, whereas ARGAQ takes 12 generations to find the feasible path and GLBR takes more than 14 generations to find the feasible path. This result shows that GLBR and ARGAQ require more genetic operations to find the feasible path. Therefore, it can be concluded that the search efficiency of QOSGA is better than GLBR and ARGAQ. As the search efficiency is better it is intuitive that the time to find a feasible path should be minimum in QOSGA compared to GLBR and ARGAQ. Table $\mathrm{V}$ gives a comparison of GA-based QoS routing algorithms.

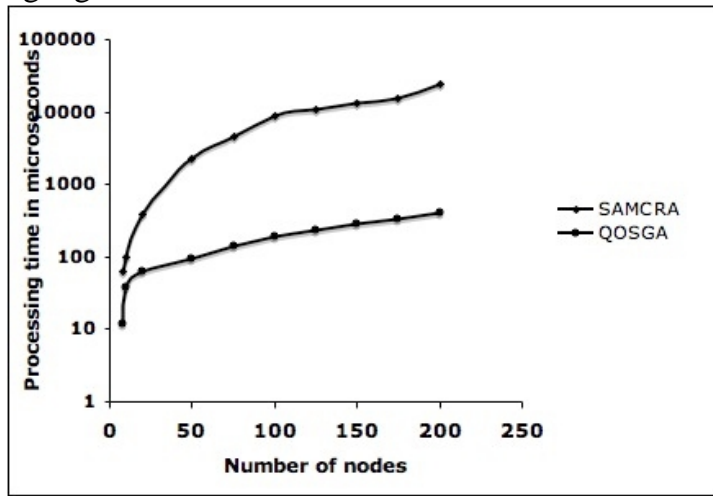

Fig. 5 Number of nodes Vs Processing time in microseconds

Table V COMPARISON OF GA-BASED ROUTING ALGORITHMS 


\begin{tabular}{|l|l|l|l|l|}
\hline Method & $\begin{array}{l}\text { Routing } \\
\text { Parameter(s) }\end{array}$ & $\begin{array}{l}\text { Gene } \\
\text { Coding }\end{array}$ & $\begin{array}{l}\text { Routing } \\
\text { Strategy }\end{array}$ & $\begin{array}{l}\text { Routing } \\
\text { Selection } \\
\text { Criterion } \\
\text { metric }\end{array}$ \\
\hline GLBR & Delay Time & $\begin{array}{l}\text { Network } \\
\text { Nodes }\end{array}$ & Source & $\begin{array}{l}\text { Single } \\
\text { Metric }\end{array}$ \\
\hline ARGA & Delay Time & $\begin{array}{l}\text { Tree } \\
\text { Junctions }\end{array}$ & Source & $\begin{array}{l}\text { Single } \\
\text { Metric }\end{array}$ \\
\hline ARGAQ & $\begin{array}{l}\text { Delay Time, } \\
\text { Transmission } \\
\text { Success Ratio }\end{array}$ & $\begin{array}{l}\text { Tree } \\
\text { Junctions }\end{array}$ & Source & $\begin{array}{l}\text { Single } \\
\text { Mixed } \\
\text { Metric }\end{array}$ \\
\hline QOSGA & $\begin{array}{l}\text { Additive } \\
\text { Metrics }\end{array}$ & $\begin{array}{l}\text { Tree } \\
\text { Junctions }\end{array}$ & Source & $\begin{array}{l}\text { Single } \\
\text { Metric }\end{array}$ \\
\hline
\end{tabular}

\section{Summary}

In this paper the need for QoS routing, existing methods for non GA based QoS routing, and GA based QoS routing have been discussed. It is observed that processing time taken by the existing non GA based QoS routing algorithms for finding a feasible path is more. This can be reduced by applying Genetic Algorithm techniques to find a feasible path. This has been proved by comparing the performance of QOSGA with that of SAMCRA in Figure 5. Also the existing GA based heuristics consider only a single or single mixed constraint but not multiple constraints. This issue of satisfying multiple constraints has been addressed by QoS routing using Genetic Algorithm (QOSGA) has been proposed. QOSGA gives the feasible path satisfying the multiple QoS constraints requirement of the application. Also QOSGA's search efficiency have been compared with existing GA based algorithms such as GLBRQ and ARGAQ in terms of the number of generation taken to compute a feasible path. From the results it has been found that QOSGA with multiple constraints performs better than the existing algorithms. Also Table $\mathrm{V}$ gives the benefits of QOSGA over other routing algorithms using GA.

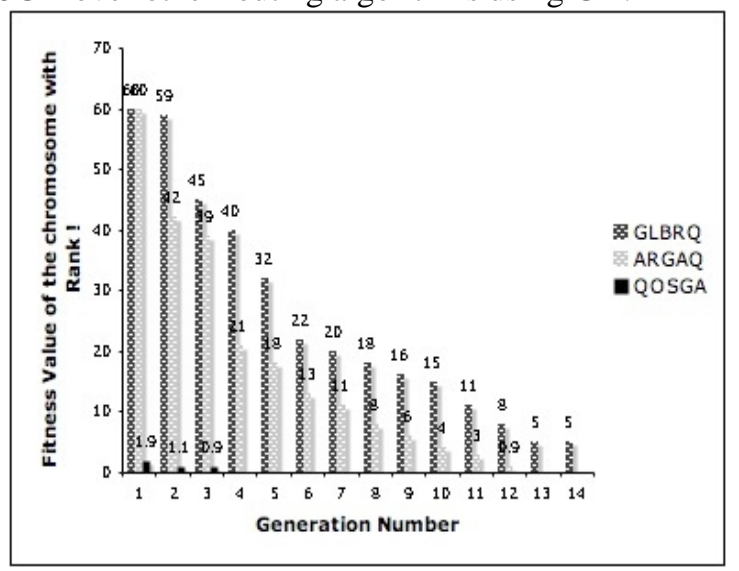

Fig. 5 Fitness value comparison Vs Generation Number taken

\section{REFERENCES}

[1] T. Korkmaz and M. Krunz, "Routing Multimedia Traffic with QoS guarantees", IEEE Trans. Multimedia, Vol.5, NO.3, 429-443, Sep. 2003.

[2] P. Van Mieghem and F.A Kuipers, "Concepts of Exact Quality of Service algorithms", IEEE Trans. NET, vol.5, NO.3, 851-864, 2004.

[3] S.Chen and K. Nahrstedt, "On finding multi-constrained paths", ICC98, IEEE New York, 874-879, 1998.
[4] F. Kupiers, T. Korkmaz, M. Krunz, and P. Van Mieghem, “A Performance Evaluation of constraint based path selection Algorithms", IEEE Trans. NET, Sep. 2004. H.De. Neve and P. Van Meighem, "TAMCRA: A Tunable Accuracy multiple constraints routing Algorithm", Computer Communications, vol.23, 667- 679, 2000.

[5] Yuchun Guo, Fernando Kuipers, and Piet Van Mieghem, "Link-disjoint paths for reliable QoS routing", International Journal of Communication Systems, vol.16, 779-798, 2003.

[6] Iwata and et. al., "ATM routing algorithms with multiple QoS requirements for multimedia Internetworking", IEICE Transactions and Communications, vol.E79-B, No.8, 999-1006, 1996.

[7] Alp'ar J'uttner, Balazs Szviatovszki, Ildik'o M'ecs, and Zsolt Rajk'o, "Lagrange Relaxation Based Method for the QoS Routing Problem", IEEE INFOCOM, 859-868, 2001.

[8] D.E.Goldberg, "Genetic Algorithms in Search, Optimization, and Machine Learning", Addison-Wesley, 1989.

[9] Leonard Barolli, Akio Koyama, Hiroto Sawada, Takuo Suganuma and Norio Shiratori, "A New QoS Routing Approach for Multimedia Applications based on Genetic Algorithms", IEEE CW, 289-295, 2002.

[10] L.Barolli, A.Koyama, T.Suganuma, and N.Shiratori, "A Genetic Algorithm Based QoS Routing Method for Multimedia Communications over High-Speed Networks", IPSJ, 544-552, 2003.

[11] Leonard Barolli, Akio Koyama, Kazunori Matsumoto, and Bernady O. Apduhan, "GA-based Multi-purpose Optimization Algorithm for QoS Routing", AINA(1), 23-28, 2004.

[12] Munetomo.M, Takai.Y, and Sato.Y, "An Adaptive Routing Algorithm with Load Balancing by a Genetic Algorithm”, IPSJ, 219-227, 1998.

[13] Xiang.F, Junzhou.L, Jieyi.W and Guanqun.G, "QoS routing based on genetic algorithm", Computer Communications, 1392-1399, 1999.

[14] Gang Cheng, Ye Tian, and Nirwan Ansari, "A New QoS Framework for Solving MCP", IEICE Transaction Communication, 534-541, 2003.

[15] Wang Z and Crowcroft J, "Quality of Service routing for supporting multimedia applications", JSAC, 1228-1234, 1996.

[16] J.C.Bean, "Genetic Algorithms and random keys for sequencing and Optimization”, ORSA J. Computing,154-160.

R.Leela obtained her degree in Electronics and Communication Engineering in 1986 from Regional Engineering College (REC), Tiruchirappalli, and Postgraduate degree in Computer Science and Engineering in 1989 from Regional Engineering College (REC), Tiruchirappalli. She is currently doing research in National Institute of Technology, Tiruchirappalli. Since 1989, she has been in the teaching profession and currently she is a faculty in the Department of Computer Science and Engineering, National Institute of Technology, Tiruchirappalli, Tamil Nadu, India. Her research interests include QoS routing, multimedia communication, Adhoc networks, and network computing.

S.Selvakaumar obtained his degree in Electronics and Com- munication Engineering with Distinction in 1983 from Madurai Kamaraj University, Madurai and Postgraduate degree in Computer Science and Engineering in 1987 from Regional Engineering College (REC), Tiruchirappalli. He was awarded Ph.D. degree by the Indian Institute of Technology Madras, Chennai in 1999. Since 1983, he has been in the teaching profession and currently he is a Professor in the Department of Computer Science and Engineering, National Institute of Technology, Tiruchirappalli, Tamil Nadu, India. His research interests include group communication in high-speed networks, routing, multimedia communication, scheduling for QoS guarantee, mobile networks, and network computing. 